\title{
TOEPLITZ OPERATORS ON CERTAIN WEAKLY PSEUDOCONVEX DOMAINS
}

\author{
DAVID CROCKER and IAIN RAEBURN
}

(Received 4 March 1980)

Communicated by G. Brown

\begin{abstract}
Let $\Omega$ be the weakly pseudoconvex domain

$$
\left\{\left(z_{1}, \ldots, z_{n}\right) \in \mathbf{C}^{n}:\left|z_{1}\right|^{2 p_{1}}+\cdots+\left|z_{n}\right|^{2 p_{n}}<1\right\},
$$

and let $\partial \Omega$ be its boundary. If $\varphi \in L^{\infty}(\partial \Omega)$, we denote by $T_{\varphi}$ the Toeplitz operator with symbol $\varphi$ acting on the Hardy space $H^{2}(\partial \Omega)$, and by $\mathscr{T}(\partial \Omega)$ the $C^{*}$-subalgebra of $B\left(H^{2}(\partial \Omega)\right.$ ) generated by the Toeplitz operators with continuous symbol. Our main theorem asserts that $\mathscr{T}(\partial \Omega)$ contains the ideal $K$ of all compact operators on $H^{2}(\partial \Omega)$, and that the symbol map $\varphi \rightarrow T_{\varphi}$ induces an isomorphism of $C(\partial \Omega)$ onto the quotient $C^{*}$-algebra $\mathscr{T}(\partial \Omega) / K$. Similar results have been established before for other domains, and in particular when $\Omega$ is strongly pseudoconvex. The main interest of our results lies in their proofs: ours are elementary, whereas those used in the strongly pseudoconvex case depend heavily on the theory of the tangential Cauchy-Riemann operator.
\end{abstract}

1980 Mathematics subject classification (Amer. Math. Soc.): primary 47 B 35; secondary 32 F 15 , $46 \mathrm{~L} 05$.

Let $\Omega$ be a bounded domain in $C^{n}$ with smooth boundary $\partial \Omega$, and let $L^{2}(\partial \Omega)$ be the space of functions on $\partial \Omega$ which are square integrable with respect to the usual surface measure on $\partial \Omega$. The smooth functions on $\partial \Omega$ which extend to be holomorphic in $\Omega$ span a closed subspace $H^{2}(\partial \Omega)$ of $L^{2}(\partial \Omega)$; we denote by $P$ the orthogonal projection of $L^{2}(\partial \Omega)$ onto $H^{2}(\partial \Omega)$. If $\varphi \in L^{\infty}(\partial \Omega)$ the Toeplitz operator with symbol $\varphi$ is the bounded operator on $H^{2}(\partial \Omega)$ defined by $T_{\Psi}(f)=$ $P(\varphi f)$. These operators have been studied in several special cases: notably, when $\Omega$ is the unit disc in $\mathbf{C}$, when $\Omega$ is the unit ball in $\mathbf{C}^{n}$, and when $\Omega$ is strongly pseudoconvex (references are given in, for example, Jewell and Krantz (1979)).

OCopyright Australian Mathematical Society 1981 
We shall consider Toeplitz operators on the domain

$$
\Omega=\left\{\left(z_{1}, \ldots, z_{n}\right) \in \mathbf{C}^{n}: \sum_{i=1}^{n}\left|z_{i}\right|^{2 p_{i}}<1\right\},
$$

where $p=\left(p_{1}, \ldots, p_{n}\right)$ is an $n$-tuple of positive integers. If some $p_{i}$ is greater than 1 , these domains are pseudoconvex, but are not strongly pseudoconvex at points of the boundary where $z_{i}$ vanishes (see, for example, D'Angelo (1978), page 260). Two of these domains are biholomorphically equivalent only if the corresponding $p_{i}$ 's are the same up to permutation (see, for example, Webster (1979), section 6). We shall suppose $n>1$ to avoid trivialities.

Our main result (Theorem 2.1) concerns the closed subalgebra $\sigma(\partial \Omega)$ of $B\left(H^{2}(\partial \Omega)\right)$ generated by $\left\{T_{\varphi}: \varphi \in C(\partial \Omega)\right\}$. It asserts that $\mathcal{T}(\partial \Omega)$ contains the ideal $K\left(H^{2}(\partial \Omega)\right)$ of compact operators, and that the quotient $C^{*}$-algebra $\sigma / K\left(H^{2}\right)$ is isomorphic to $C(\partial \Omega)$. This result is known for other choices of the domain $\Omega$ : when $\Omega$ is the unit ball (that is, when each $p_{i}=1$ ) it appears in Coburn (1973), and it was proved for general strongly pseudoconvex domains (and $n>2$ ) by Raeburn (1978), and by Sato and Yabuta (1978). The crux of the argument in this last case is to show that for $\varphi \in C(\partial \Omega)$ the operator $(I-P) M_{\varphi}: H^{2} \rightarrow L^{2}$ is compact (where $M_{\varphi}$ stands for multiplication by $\varphi$ ); the proof of this uses the theory of the tangential Cauchy-Riemann operator $\bar{\partial}_{b}$ (see Folland and Kohn (1972)). This theory is not available in our setting; since the Levi form of $\Omega$ has zero eigenvalues at points of $\partial \Omega$ where $z_{i}=0$ and $p_{i}>1$ (D'Angelo (1978), top of p. 260), the domain does not satisfy condition $Y(1)$ of Folland and Kohn (1972), p. 94. The compactness of $(I-P) M_{\varphi}$ is used to show that the commutators $T_{\varphi} T_{\psi}-T_{\psi} T_{\varphi}$ of Toeplitz operators are compact; we do it here by calculating matrix entries relative to a fixed basis for $H^{2}(\partial \Omega)$.

In fact it turns out to be easier to do the necessary calculations for Toeplitz operators defined in the interior, so we do this first in section 1 . We then show that the two notions of Toeplitz operators are the same up to compact perturbation, and hence deduce our main theorem from the corresponding result in the interior (section 2). Our third section contains a few remarks on some related results which fall out without much extra effort; in particular, we consider Toeplitz operators with other than continuous symbols.

Throughout this paper, $\Omega$ will be a fixed domain of the type introduced above, and $\partial \Omega$ will be its boundary. We shall use the usual multi-index notation: thus $z^{I}=z_{1}^{i_{1}} \cdots z_{n}^{i_{n}}$ and $|I|=\sum i_{j}$. We shall write $e_{r}$ for the multi-index with a 1 in the $i$ th slot and 0 's elsewhere, so that, for example, $I+e_{1}=\left(i_{1}+1, i_{2}, \ldots, i_{n}\right)$. If $H$ is a Hilbert space, $B(H)$ will be the algebra of all bounded operators on $H$ and $K(H)$ the ideal of compact operators. The Hilbert spaces $L^{2}(\Omega)$ and $L^{2}(\partial \Omega)$ are those associated to the usual Lebesgue measure $d V$ on $\Omega$ and surface measure $d S$ on $\partial \Omega$. 


\section{Toeplitz operators acting in the interior}

We denote by $H^{2}(\Omega)$ the closed subspace of $L^{2}(\Omega)$ consisting of those functions which are holomorphic in $\Omega$, and we let $Q: L^{2}(\Omega) \rightarrow H^{2}(\Omega)$ be the orthogonal projection. If $\varphi \in C(\bar{\Omega})$ then the Toeplitz operator $S_{\varphi}$ with symbol $\varphi$ is the bounded operator on $H^{2}(\Omega)$ defined by $S_{\varphi}(f)=Q(\varphi f)$. We let $\circlearrowleft(\Omega)$ be the $C^{*}$-subalgebra of $B\left(H^{2}(\Omega)\right)$ generated by $\left\{S_{\varphi}: \varphi \in C(\bar{\Omega})\right\}$. This section is devoted to proving the following theorem:

THEOREM 1.1. The $C^{*}$-algebra $\mathcal{T}(\Omega)$ contains the ideal $K\left(H^{2}(\Omega)\right)$ and the map $\left.S_{\varphi} \rightarrow \varphi\right|_{\partial \Omega}$ induces an isomorphism of $\mathcal{T}(\Omega) / K\left(H^{2}\right)$ onto $C(\partial \Omega)$.

We prove that $\sigma(\Omega) \supset K\left(H^{2}\right)$ by the standard technique (see Douglas (1972a), Theorem 5.39) of showing that $\mathscr{T}(\Omega)$ is irreducible and contains nonzero compact operators. The argument used in the first part of Raeburn (1978), Theorem 2.2 shows that $\mathscr{T}(\Omega)$ is irreducible, and we shall see later that there are plenty of non-zero compact operators of the form $S_{\varphi} S_{\psi}-S_{\varphi \psi}$.

The isomorphism $\xi: \mathcal{T} / K \rightarrow C(\partial \Omega)$ is defined by $\xi\left(S_{\varphi}+K\right)=\varphi$ las; to show that $\xi$ is well-defined we need to prove ${ }^{1}$ that if $S_{\varphi}$ is compact then $\left.\varphi\right|_{\partial \Omega}$ is identically zero. This will follow from the argument used in the proof of Raeburn (1978), Theorem 2.3, if we can construct holomorphic functions which peak at points of $\partial \Omega$. One way to do this is to observe that if $w \in \partial \Omega$, then

$$
f_{w}(z)=\frac{1}{2-\sum_{i} z_{i}^{p_{i}} \bar{w}_{i}^{p_{i}}}
$$

satisfies $\left|f_{w}(z)\right| \leqslant 1$ on $\bar{\Omega}$, and $f_{w}(z)=1$ only if $z_{i}^{p_{i}}=w_{i}^{p_{i}}$, so that $f_{w}$ peaks locally at $w \in \partial \Omega$, and then to use the construction of Hakim and Sibony (1977), Rémarque 2 to obtain a global peak function. The map $\xi$ is *-linear, is clearly onto, and is injective by (a slight generalization of) Theorem 2.3 of Venugopalkrishna (1972), so all that remains is to show that $\xi$ is a homomorphism.

Thus we have to prove that $S_{\varphi} S_{\psi}-S_{\varphi \psi}$ is compact for each $\varphi, \psi \in C(\bar{\Omega})$. By the Stone-Weierstrass theorem and the linearity of the map $\varphi \rightarrow S_{\varphi}$ we need only consider $\varphi$ and $\psi$ of the form $z \bar{z}^{J}$, and some elementary manipulations show it is enough to prove that each operator of the form

$$
S_{z_{r}} S_{\bar{z}_{s}}-S_{z_{r} \bar{z}_{s}}
$$

is compact (and since these are not zero, this will also complete the proof that $\sigma(\Omega) \supset K\left(H^{2}\right)$ ). We shall prove these are compact by calculating their matrix entries in a suitable orthonormal basis.

${ }^{1}$ This is not enough. It is more satisfactory to define $\eta=C(\partial \Omega) \rightarrow \mathcal{J} / K$ by $\eta(\varphi)=S_{\psi}$ $+K$, where $\psi \in C(\bar{\Omega})$ satisfies $\left.\psi\right|_{\partial \Omega}=\varphi$; the properties of the $S_{\varphi}$ established here, used in a different order, show that $\eta$ is well-defined and an isomorphism. 
We shall parametrize $\Omega$ by

$$
\Omega=\left\{\left(\gamma_{i}^{1 /\left(2 p_{i}\right)} e^{i \theta_{i}}\right)_{i=1}^{n}: \gamma_{i} \in[0,1], \theta_{i} \in[0,2 \pi], \sum \gamma_{i}<1\right\}
$$

the volume measure on $\Omega$ is then given by

$$
d V=\left(\prod_{i=1}^{n} \frac{1}{2 p_{i}} \gamma^{\left(1 / p_{i}\right)-1}\right) d \gamma_{1} \cdots d \gamma_{n} d \theta_{1} \cdots d \theta_{n}
$$

It is easy to see that $\left\{z^{I}: I>0\right\}$ is an orthogonal set in $H^{2}(\Omega)$, and since $\Omega$ is polynomially convex, the Oka-Weil theorem implies that they span $H^{2}(\Omega)$. Hence an orthonormal basis for $H^{2}(\Omega)$ is given by $\left\{e^{I}: I>0\right\}$ where $e^{I}=$ $z^{I} /\left\|z^{I}\right\|$. Simple calculations show that

$$
S_{z_{r}}\left(e^{I}\right)=\frac{\left\|z^{I+e_{r}}\right\|}{\left\|z^{I}\right\|} e^{I+e_{r}}
$$

and, since $\bar{z}_{r} z^{I}$ is orthogonal to every $z^{J}$ except $z^{I-e}$,

$$
S_{\bar{z}_{r}}\left(e^{I}\right)=\left\{\begin{array}{l}
\frac{1}{\left\|z^{I}\right\|}\left(\bar{z}_{r} z^{I}, \frac{1}{\left\|z^{I-e_{r}}\right\|} z^{I-e_{r}}\right) e^{I-e_{r}}=\frac{\left\|z^{I}\right\|}{\left\|z^{I-e_{r}}\right\|} e^{I-e_{r}} \quad\left(i_{r}>0\right), \\
0 \quad\left(i_{r}=0\right) .
\end{array}\right.
$$

Similarly we can compute $S_{z, \bar{z}_{s}}$, and if $s \neq r$ we obtain

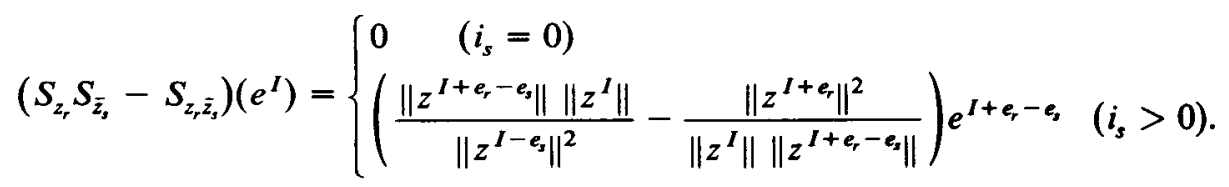

Thus to prove that $S_{z_{r}} S_{\bar{z}_{s}}-S_{z_{r} \bar{z}_{s}}$ is compact in the case $s \neq r$, we have to show that

$$
\lambda(I)=\frac{\left\|z^{I+e_{r}-e_{s}}\right\|\left\|z^{I}\right\|}{\left\|z^{I-e_{s}}\right\|^{2}}-\frac{\left\|z^{I+e_{r}}\right\|^{2}}{\left\|z^{I}\right\|\left\|z^{I+e_{r}-e_{s}}\right\|} \rightarrow 0 \quad \text { as }|I| \rightarrow \infty .
$$

For $n=2$ the following elementary lemma is a well-known property of the beta function; the general result can be deduced from the $n=2$ case by induction.

LEMMA 1.2. Let $\Delta_{n}=\left\{\left(\gamma_{1}, \ldots, \gamma_{n}\right): \gamma_{i} \in[0,1]\right.$ and $\left.\Sigma_{i} \gamma_{i}<1\right\}$, and suppose that $\alpha_{j}>-1$ for $j=1, \ldots, n$. Then

$$
\int_{\Delta_{n}}\left(\prod_{j=1}^{n} \gamma_{j}^{\alpha_{j}}\right) d \gamma_{1} \cdots d \gamma_{n}=\frac{\prod_{j=1}^{n} \Gamma\left(\alpha_{j}+1\right)}{\Gamma\left(n+1+\sum_{j=1}^{n} \alpha_{j}\right)}
$$


Corollary 1.3 (D'Angelo (1978), Lemma 2).

$$
\left\|z^{I}\right\|^{2}=\frac{\pi^{n}}{|p|}\left(\prod_{j=1}^{n} \Gamma\left(\frac{i_{j}+1}{p_{j}}\right)\right) / \Gamma\left(\sum_{j=1}^{n} \frac{i_{j}+1}{p_{j}}+1\right) .
$$

After a bit of simplification, it follows that

$$
\begin{aligned}
|\lambda(I)|= & {\left[\frac{\Gamma\left(a+\frac{1}{p_{s}}\right) \Gamma\left(a+\frac{1}{p_{r}}\right) \Gamma\left(\frac{i_{r}+2}{p_{r}}\right) \Gamma\left(\frac{i_{s}+1}{p_{s}}\right)}{\Gamma\left(a+\frac{1}{p_{r}}+\frac{1}{p_{s}}\right)^{2} \Gamma\left(\frac{i_{r}+1}{p_{r}}\right) \Gamma\left(\frac{i_{s}}{p_{s}}\right)}\right]^{1 / 2} } \\
& \cdot\left|\frac{\Gamma(a) \Gamma\left(a+\frac{1}{p_{r}}+\frac{1}{p_{s}}\right)}{\Gamma\left(a+\frac{1}{p_{r}}\right) \Gamma\left(a+\frac{1}{p_{s}}\right)}-1\right|
\end{aligned}
$$

where we have set

$$
a=\left(\sum_{j=1, j \neq s}^{n} \frac{i_{j}+1}{p_{j}}\right)+\frac{i_{s}}{p_{s}}+1
$$

Thus

$$
|\lambda(I)|=\left[\frac{\beta\left(b+\frac{1}{p_{r}}, c\right) \beta\left(d+\frac{1}{p_{s}}, e\right)}{\beta(b, c) \beta(d, e)}\right]^{1 / 2}|\mu(I)-1|,
$$

where we have taken

$$
\begin{aligned}
& b=\frac{i_{r}+1}{p_{r}}, \quad c=\sum_{j \neq r} \frac{i_{j}+1}{p_{j}}+1, \\
& d=\frac{i_{s}}{p_{s}}, \quad e=\sum_{j \neq s} \frac{i_{j}+1}{p_{j}}+1+\frac{1}{p_{r}} .
\end{aligned}
$$

Since the beta function is decreasing in either variable, we deduce that

$$
|\lambda(I)| \leqslant|\mu(I)-1| \text { for } I \geqslant 0 .
$$

Now if $x \geqslant 0$, then from Erdélyi (1953), p. 47, we have

$$
\frac{\Gamma(x+\alpha)}{\Gamma(x+\beta)}=x^{\alpha-\beta}+O\left(x^{\alpha-\beta-1}\right)
$$

thus $\mu(I) \rightarrow 1$ as $a \rightarrow \infty$, or, equivalently, $\lambda(I) \rightarrow 0$ as $|I| \rightarrow \infty$. 
It still remains to consider the case $s=r$; without loss of generality we suppose $r=1$. Then

$$
\left(S_{z_{r}} S_{\bar{z}_{r}}-S_{z, \bar{z}_{r}}\right)\left(e^{I}\right)= \begin{cases}-\frac{\left\|z^{I+e_{1}}\right\|^{2}}{\left\|z^{I}\right\|^{2}} e^{I} & \left(i_{1}=0\right), \\ \left(\frac{\left\|z^{I}\right\|^{2}}{\left\|z^{I-e_{1}}\right\|^{2}}-\frac{\left\|z^{I+e_{1}}\right\|^{2}}{\left\|z^{I}\right\|^{2}}\right) e^{I} & \left(i_{1}>0\right) .\end{cases}
$$

We deal with the $i_{1}=0$ case first: now

$$
\frac{\left\|z^{I+e_{1}}\right\|^{2}}{\left\|z^{I}\right\|^{2}}=\frac{\Gamma\left(\frac{2}{p_{1}}\right) \Gamma(a)}{\Gamma\left(\frac{1}{p_{1}}\right) \Gamma\left(a+\frac{1}{p_{1}}\right)}
$$

where as before we have taken

$$
a=\sum_{j \neq 1} \frac{i_{j}+1}{p_{j}}+\frac{1}{p_{1}}+1
$$

The right-hand side of (1) goes to 0 as $|I| \rightarrow \infty$ since it behaves like $a^{-1 / p_{1}}$. If $i_{1}>0$, then

$$
\begin{aligned}
\left(S_{z_{r}} S_{\bar{z}_{r}}-S_{\left|z_{r}\right|^{2}}\right)\left(e^{I}\right) & =\left[\frac{\Gamma\left(\frac{i_{1}+1}{p_{1}}\right) \Gamma(c)}{\Gamma\left(\frac{i_{1}}{p_{1}}\right) \Gamma\left(c+\frac{1}{p_{1}}\right)}-\frac{\Gamma\left(\frac{i_{1}+2}{p_{1}}\right) \Gamma\left(c+\frac{1}{p_{1}}\right)}{\Gamma\left(\frac{i_{1}+1}{p_{1}}\right) \Gamma\left(c+\frac{2}{p_{1}}\right)}\right] e^{I} \\
& =\left[\frac{\beta\left(a+\frac{1}{p_{1}}, b\right)}{\beta(a, b)}-\frac{\beta\left(a+\frac{2}{p_{1}}, b\right)}{\beta\left(a+\frac{1}{p_{1}}, b\right)}\right] e^{I} \\
& =\left[S(a, b)-S\left(a+\frac{1}{p_{1}}, b\right)\right] e^{I},
\end{aligned}
$$

where we have written

$$
\begin{gathered}
c=\sum_{j \neq 1} \frac{i_{j}+1}{p_{j}}+\frac{i_{1}}{p_{1}}+1, \quad a=\frac{i_{1}}{p_{1}}, \\
b=c-a, \quad S(x, y)=\frac{\beta\left(x+\frac{1}{p_{1}}, y\right)}{\beta(x, y)} .
\end{gathered}
$$


A simple calculation shows that (writing $\varepsilon=1 / p_{1}$ )

$$
\begin{gathered}
S(a, b)-S(a+\varepsilon, b)=\frac{\int_{0}^{1} \int_{0}^{1}(\rho \sigma)^{a-1}[(1-\rho)(1-\sigma)]^{b-1}\left[(\sigma \rho)^{e}-\sigma^{2 \varepsilon}\right] d \rho d \sigma}{\beta(a, b) \beta(a+\varepsilon, b)} \\
=\frac{-1}{\beta(a, b) \beta(a+\varepsilon, b)} \iint_{\rho<\sigma}(\rho \sigma)^{a-1}[(1-\rho)(1-\sigma)]^{b-1}\left[\sigma^{\varepsilon}-\rho^{e}\right]^{2} d \rho d \sigma,
\end{gathered}
$$

which is clearly negative. Thus since $p_{1}>1$ we have

$$
\left|S(a, b)-S\left(a+\frac{1}{p_{1}}, b\right)\right| \leqslant|S(a, b)-S(a+1, b)| .
$$

Now expanding $S$ in gamma functions shows that

$$
S(a+1, b)=\left(1+\frac{1}{p_{1} a}\right) S(a, b) /\left(1+\frac{1}{p_{1}(a+b)}\right),
$$

and so

$$
|S(a, b)-S(a+1, b)|<S(a, b) \frac{b}{p_{1} a(a+b)} .
$$

Using the estimate $\Gamma(a+\varepsilon) / \Gamma(a) \sim a^{e}$ we see that

$$
|S(a, b)-S(a+1, b)|<C\left(\frac{a}{a+b}\right)^{1 / p_{1}} \frac{b}{p_{1} a(a+b)},
$$

which converges to zero by the following trivial lemma:

LEMMA 1.4. Suppose that $a, b>\varepsilon>0, \alpha>0$. Then

$$
\left(\frac{a}{a+b}\right)^{\alpha}\left[\frac{b}{a(a+b)}\right] \rightarrow 0 \text { as } a+b \rightarrow \infty \text {. }
$$

The proof of Theorem 2.1 is now complete.

\section{Toeplitz operators acting on the boundary}

In this section we prove the following theorem (with notation as in the Introduction):

THEOREM 2.1. The $C^{*}$-algebra $\mathcal{T}(\partial \Omega)$ contains the ideal $K\left(H^{2}(\partial \Omega)\right)$ and the symbol map $\varphi \rightarrow T_{\varphi}$ induces an isomorphism of $C(\partial \Omega)$ with $\mathscr{T}(\partial \Omega) / K\left(H^{2}\right)$.

We want to use the proof of Theorem 2.2 of Raeburn (1978). Since we have already observed in the course of proving Theorem 2.1 that there are holomorphic peak functions for the points of $\partial \Omega$, all we have to do to make this proof 
work is to show that operators of the form $T_{\varphi} T_{\psi}-T_{\varphi \psi}$ are compact. We shall do this indirectly by showing that there is a unitary operator $U: H^{2}(\partial \Omega) \rightarrow$ $H^{2}(\Omega)$ such that for each $\psi \in C(\bar{\Omega})$ the operator

$$
U^{*} S_{\psi} U-T_{\psi \mid}
$$

is compact; the result follows easily from this, Theorem 2.1 and the observation that each $\psi \in C(\partial \Omega)$ is the restriction to $\partial \Omega$ of some $\psi \in C(\bar{\Omega})$. This approach also yields the following result, which is still an open question for arbitrary strongly pseudoconvex domains (see the concluding remarks in Raeburn (1978)).

CoROllary 2.2. The $C^{*}$-algebras $\mathcal{T}(\Omega)$ and $\mathcal{T}(\partial \Omega)$ are equivalent extensions of $C(\partial \Omega)$ by the ideal of compact operators.

We shall use the parametrisation

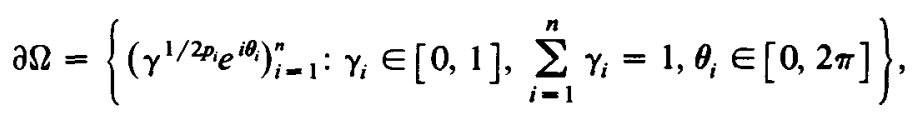

so that the free parameters are $\gamma_{1}, \ldots, \gamma_{n-1}$ and $\theta_{1}, \ldots, \theta_{n}$. The surface area measure on $\partial \Omega$ is given by

$$
d S=\frac{1}{2^{n-1}|p|}\left(\prod_{i=1}^{n} \gamma_{i}^{\left(1 / p_{i}\right)-1}\right)\left(\sum_{i=1}^{n} p_{i}^{2} \gamma^{\left(2-\left(1 / p_{i}\right)\right)}\right)^{1 / 2} d \gamma_{1} \cdots d \gamma_{n-1} d \theta_{1} \cdots d \theta_{n} .
$$

Again, it is clear that the $z^{I}$ are othogonal in $H^{2}(\partial \Omega)$, and since $\Omega$ is polynomially convex they form a basis. We therefore take as an orthonormal basis for $H^{2}(\partial \Omega)$ the set $\left\{f^{I}=z^{I} /\left\|z^{I}\right\|_{\partial \Omega}: I>0\right\}$. (To distinguish between the norms of $z^{I} \in H^{2}(\partial \Omega)$ and $z^{I} \in H^{2}(\Omega)$ we shall sometimes write $\left\|z^{I}\right\|_{\partial \Omega}$ for the former and $\left\|z^{I}\right\|_{\Omega}$ for the latter.) We write $\gamma^{\prime}$ for $\left(\gamma_{1}, \ldots, \gamma_{n-1}\right), \gamma_{n}=1-\Sigma_{i<n-1} \gamma_{i}$, and set

$$
\tau(\gamma)=\left[\sum_{i=1}^{n} p_{i}^{2} \gamma_{i}^{2-\left(1 / p_{i}\right)}\right]
$$

We note that $\tau$ is continuous on the set $\Delta_{n-1}=\left\{\gamma^{\prime}: \Sigma \gamma_{i}<1\right\}$, and that $\tau$ does not vanish on $\Delta_{n-1}$; hence there are positive constants $\varepsilon$ and $M$ such that $\varepsilon<\tau\left(\gamma^{\prime}\right)<M$ for $\gamma^{\prime} \in \Delta_{n-1}$.

The unitary operator $U: H^{2}(\partial \Omega) \rightarrow H^{2}(\Omega)$ is defined by $U\left(f^{f}\right)=e^{I}$. An application of the Stone-Weierstrass theorem shows that it is enough for us to check that the operators $U^{*} S_{z_{i}} U-T_{z_{i}}$ are compact; without loss of generality we shall suppose $i=1$. Calculations show that

$$
\left(U^{*} S_{z_{i}} U-T_{z_{i}}\right)\left(f^{I}\right)=\left(\frac{\left\|z^{I+e_{1}}\right\|_{\Omega}}{\left\|z^{I}\right\|_{\Omega}}-\frac{\left\|z^{I+e_{1}}\right\|_{\partial \Omega}}{\left\|z^{I}\right\|_{\partial \Omega}}\right) f^{I+e_{1}}
$$


If we set

$$
A(I)=\frac{\left\|z^{I+e_{1}}\right\|_{\Omega}}{\left\|z^{I}\right\|_{\Omega}}, \quad B(I)=\frac{\left\|z^{I+e_{1}}\right\|_{\partial \Omega}}{\left\|z^{I}\right\|_{\partial \Omega}},
$$

then we have to prove that $A(I)-B(I) \rightarrow 0$ as $|I| \rightarrow \infty$. We note that $A-B=$ $\left(A^{2}-B^{2}\right) /(A+B)$, and that, in the notation of Section $1, A=S(a, b+1)^{1 / 2}$ where

$$
a=\frac{i_{1}+1}{p_{1}}, \quad b=\sum_{j=2}^{n} \frac{i_{j}+1}{p_{j}} .
$$

The following lemma is easily verified by induction:

\section{LEMMA 2.3.}

$$
\int_{\Delta_{n-1}} \prod_{j=1}^{n} \gamma_{j}^{\alpha_{j}} d \gamma^{\prime}=\frac{\prod_{j=1}^{n} \Gamma\left(\alpha_{j}+1\right)}{\Gamma\left(\sum_{j=1}^{n} \alpha_{j}+n\right)} \quad\left(\alpha_{j}>-1\right)
$$

Using this lemma and the estimates $\varepsilon<\tau<M$, we obtain

$$
B>\left(\frac{\varepsilon}{M}\right)^{1 / 2}\left(\frac{\Gamma\left(\frac{i_{1}+2}{p_{1}}\right) \Gamma\left(\sum_{j=1}^{n} \frac{i_{j}+1}{p_{j}}\right)}{\Gamma\left(\frac{i_{1}+2}{p_{1}}+\sum_{j=2}^{n} \frac{i_{j}+1}{p_{j}}\right) \Gamma\left(\frac{i_{1}+1}{p_{1}}\right)}\right)^{1 / 2}=\left(\frac{\varepsilon}{M}\right)^{1 / 2} S(a, b)^{1 / 2},
$$

and so

$$
A+B>S(a, b+1)^{1 / 2}+\left(\frac{\varepsilon}{M}\right)^{1 / 2} S(a, b)^{1 / 2}
$$

Now

$$
S(a, b+1)=\frac{a+b}{a+b+\left(1 / p_{1}\right)} S(a, b)>\frac{1}{2} S(a, b)
$$

for $a+b$ sufficiently large, and so for large $a+b$ we have

$$
|A-B|<\frac{1}{C S(a, b)^{1 / 2}}\left|A^{2}-B^{2}\right|
$$


An application of the second mean value theorem for integrals (to the denominator) shows that there is some $\delta \in[\varepsilon, M]$ such that

$$
\begin{aligned}
S(a, b)^{-1 / 2}\left(A^{2}-B^{2}\right) & =\frac{\int_{\Delta_{n-1}}\left(\prod_{j=1}^{n} \gamma_{j}^{\left(\left(_{j}+1\right) / p_{j}\right)-1}\right)\left[S(a, b+1)-\gamma_{1}^{1 / p_{1}}\right] \tau\left(\gamma^{\prime}\right) d \gamma^{\prime}}{S(a, b)^{1 / 2} \delta \int_{\Delta}\left(\prod_{j=1}^{n} \gamma_{j}^{\left.\left(i_{j}+1\right) / p_{j}\right)-1}\right) d \gamma^{\prime}} \\
& =S(a, b)^{-1 / 2} \int H_{I}\left(\gamma^{\prime}\right) \tau\left(\gamma^{\prime}\right) d \gamma^{\prime},
\end{aligned}
$$

say. To show this tends to zero as $|I| \rightarrow \infty$, it is enough by the StoneWeierstrass theorem to prove that

$$
S(a, b)^{-1 / 2} \int H_{I}\left(\gamma^{\prime}\right)\left(\gamma^{\prime}\right)^{M} d \gamma^{\prime} \rightarrow 0 \quad \text { as }|I| \rightarrow \infty
$$

for every multi-index $M \in \mathbf{N}^{n-1}$, and that

$$
S(a, b)^{-1 / 2} \int\left|H_{I}\left(\gamma^{\prime}\right)\right| d \gamma^{\prime}
$$

is bounded for all $I$. But

$$
\begin{aligned}
S(a, b)^{-1 / 2} \int\left|H_{I}\left(\gamma^{\prime}\right)\right| d \gamma^{\prime} & \leqslant S(a, b)^{-1 / 2}[S(a, b+1)+S(a, b)] \\
& =S(a, b)^{1 / 2}\left[\frac{1}{1+\frac{1}{p_{1}(a+b)}}+1\right]
\end{aligned}
$$

which is clearly bounded, so it remains to verify (2). So let $M \in \mathbf{N}^{n-1}$. Then

$$
\left|\int S(a, b)^{-1 / 2} H_{I}\left(\gamma^{\prime}\right)\left(\gamma^{\prime}\right)^{M} d \gamma^{\prime}\right|=\frac{1}{\delta S(a, b)^{1 / 2}}|S(a, b+1) U-V|,
$$

where

$$
\begin{gathered}
U=\frac{\prod_{j=1}^{n-1} \Gamma\left(\frac{i_{j}+1}{p_{j}}+m_{j}\right) \Gamma(a+b)}{\prod_{j=1}^{n-1} \Gamma\left(\frac{i_{j}+1}{p_{j}}\right) \Gamma(a+b+|M|)}, \\
V=\frac{\Gamma\left(\frac{i_{1}+2}{i_{1}}+m_{1}\right) \prod_{j=2}^{n-1} \Gamma\left(\frac{i_{j}+1}{p_{j}}+m_{j}\right) \Gamma(a+b)}{\prod_{j=1}^{n-1} \Gamma\left(\frac{i_{j}+1}{p_{j}}\right) \Gamma\left(a+b+\frac{1}{p_{1}}+|M|\right)} .
\end{gathered}
$$

From the expression of $U$ as a quotient of integrals we have at once that $U<1$. 
Hence the right-hand side of (3) is less than

$$
\begin{aligned}
\frac{1}{\delta S(a, b)^{1 / 2}}\left|S(a, b+1)-\frac{V}{U}\right| & \\
= & \frac{1}{\delta S(a, b)^{1 / 2}}\left|S(a, b+1)-S\left(a+m_{1}, b+\sum_{j=2}^{n-1} m_{j}\right)\right| .
\end{aligned}
$$

It is easy to see that if $m \in \mathbf{N}$, then

$$
S(a, b+m)=\lambda_{m}(a, b) S(a, b),
$$

for some $\lambda_{m}(a, b)$ which tends to 1 as $a+b \rightarrow \infty$. Thus we still have to show that

$$
\frac{1}{\delta S(a, b)^{1 / 2}}\left|S(a, b)-S\left(a+m_{1}, b\right)\right| \rightarrow 0 \quad \text { as } a+b \rightarrow \infty .
$$

Expanding $S\left(a+m_{1}, b\right)$ shows that this is

$$
\frac{1}{\delta} S(a, b)^{1 / 2}\left|\prod_{r=0}^{m-1}\left(\frac{1+\frac{1}{p_{1}(a+r)}}{1+\frac{1}{p_{1}(a+r+b)}}\right)-1\right|
$$

which is less than

$$
\frac{1}{\delta} S(a, b)^{1 / 2}\left|\left(1+\frac{1}{p_{1} a}\right)^{m}-\left(1+\frac{1}{p_{1}(a+b+m-1)}\right)^{m}\right| .
$$

Since $\left|x^{m}-y^{m}\right| \leqslant m 2^{m}|x-y|$ for $x, y \in[0,2]$, this is bounded by

$$
\frac{m 2^{m}}{\delta} S(a, b)^{1 / 2}\left|\frac{b+m-1}{a(a+b+m-1)}\right|<C_{1} S(a, b)^{1 / 2} \frac{b}{a(a+b)} .
$$

Using the same estimate as before for $\Gamma(a+\varepsilon) / \Gamma(a)$, we see that the expression in (4) is bounded by

$$
C_{2}\left(\frac{a}{a+b}\right)^{1 / 2 p_{1}}\left(\frac{b}{a(a+b)}\right)
$$

for some constant $C_{2}$. This converges to 0 as $a+b \rightarrow \infty$ by Lemma 1.4. Thus (4) is proved and we have completed the proof of Theorem 2.1.

\section{Concluding remarks}

Toeplitz operators with discontinuous symbol

Let $\mathscr{T}\left(L^{\infty}\right)$ be the $C^{*}$-subalgebra of $B\left(H^{2}(\partial \Omega)\right)$ generated by the Toeplitz operators $T_{\varphi}$ with symbol $\varphi \in L^{\infty}(\partial \Omega)$, and let $J$ be the closed ideal in $\mathscr{T}\left(L^{\infty}\right)$ 
generated by $\left\{T_{\varphi \psi}-T_{\varphi} T_{\psi}: \varphi, \psi \in L^{\infty}\right\}$. Jewell and Krantz have shown that the map $\varphi \rightarrow T_{\varphi}$ induces an isomorphism of $L^{\infty}(\partial \Omega)$ with the quotient algebra $\mathscr{T}\left(L^{\infty}\right) / J$ (Theorem 14 of Jewell and Krantz (1979) and the subsequent remark). In the case of a strongly pseudoconvex domain, they went on to consider Toeplitz operators with symbol in the Banach algebra $H^{\infty}+C$, but they were unable to extend their results to more general domains. They did observe, however, that in our case the space $H^{\infty}(\partial \Omega)+C(\partial \Omega)$ is a closed subalgebra of $L^{\infty}(\partial \Omega)$ (Jewell and Krantz (1979), Remark 1, p. 310). The result Jewell and Krantz lacked was the following:

Proposition 3.1. If $\varphi \in C(\partial \Omega)$, then the operator $(I-P) M_{\varphi} P \in B\left(L^{2}(\partial \Omega)\right)$ is compact.

Proof (see Janas (1976b), p. 186). We have already seen in Section 2 that the operator $T_{\bar{\varphi}} T_{\varphi}-T_{|\varphi|^{2}}=P M_{\varphi}(I-P) M_{\varphi}$ is compact; thus if $f_{m} \rightarrow 0$ weakly in $H^{2}$, then

$$
\left\|(I-P) M_{\varphi} f_{m}\right\|^{2}=\left(P M_{\varphi}(I-P) M_{\varphi} f_{m}, f_{m}\right) \rightarrow 0,
$$

which proves the result. (We remark that although arguments like those used in Section 1 can be used to show that $(I-P) M_{\bar{z}_{1}}: H^{2}(\Omega) \rightarrow L^{2}(\Omega)$ is compact, we do not know how to prove directly that the corresponding operator on the boundary is compact.)

It follows from this proposition that if $\varphi \in C(\partial \Omega)$ and $\psi \in L^{\infty}(\partial \Omega)$ then $T_{\psi} T_{\varphi}-T_{\psi \phi}$ is compact. For $\varphi \in H^{\infty}(\partial \Omega)$ we have the equality $T_{\psi} T_{\Phi}=T_{\psi \phi}$, so that if $\mathscr{T}\left(H^{\infty}+C\right)$ is the subalgebra of $B\left(H^{2}\right)$ generated by the Toeplitz operators with symbol in $H^{\infty}(\partial \Omega)+C(\partial \Omega)$, then we have $J \cap \mathcal{T}\left(H^{\infty}+C\right)=$ $K\left(H^{2}\right)$. The following theorem now follows from Jewell and Krantz's result on the $C^{*}$-algebra $\mathscr{T}\left(L^{\infty}\right)$ :

THEOREM 3.2. The closed subalgebra $\mathcal{T}\left(H^{\infty}+C\right)$ of $B\left(H^{2}(\partial \Omega)\right)$ generated by $\left\{T_{\varphi}: \varphi \in H^{\infty}(\partial \Omega)+C(\partial \Omega)\right\}$ contains $K\left(H^{2}(\partial \Omega)\right)$ and the symbol map $\varphi \rightarrow$ $T_{\phi}$ induces an isomorphism of $H^{\infty}(\partial \Omega)+C(\partial \Omega)$ onto the quotient algebra $\mathscr{T}\left(H^{\infty}+C\right) / K\left(H^{2}\right)$.

\section{The index of Toeplitz operators}

Our main results imply that a Toeplitz operator $T_{\varphi}$ on $H^{2}(\partial \Omega)$ (respectively, on $H^{2}(\Omega)$ ) is Fredholm if and only if the symbol $\varphi \in C(\partial \Omega)^{-1}$ (respectively iff $\left.\left.\varphi\right|_{a \Omega} \in C(\partial \Omega)^{-1}\right)$. Since $\partial \Omega$ is simply connected, all these Fredholm operators have index 0 by the argument of Theorem 1.4 of Venugopalkrishna (1972). This is, of course, no longer the case if we consider Toeplitz operators with matrixvalued symbols. The analogues of Theorems 1.1 and 2.1 are obtained by 
tensoring the appropriate exact sequence

$$
0 \rightarrow K\left(H^{2}\right) \rightarrow \sigma \rightarrow C(\partial \Omega) \rightarrow 0
$$

by the $C^{*}$-algebra $M_{N}(\mathrm{C})$, and again we see that $T_{\varphi}$ is Fredholm precisely when the symbol $\varphi$ is invertible. Since the domain $\Omega$ is star-shaped we can use the reasoning of Janas (1976a), Corollary 2, to see that the index formula of Venugopalkrishna (1972), Theorem 1.5, applies in this case; that the same formula holds for operators defined on the boundary follows from our Corollary 2.2. This index formula can also be formulated in terms of $K$-theory (see Boutet de Monvel (1979)).

\section{Toeplitz operators in the polydisc}

We close with an example showing that commutators of Toeplitz operators defined on the boundary of a domain in $C^{n}$ need not be compact. Let $D$ be the unit polydisc in $\mathbf{C}^{2}$, so that

$$
\partial D=\left\{(z, w) \in C^{2} \text { : either }|z|=1 \text {, or }|w|=1 \text {, or both }\right\} .
$$

We then take $H^{2}(\partial D)$ to be the closed span in $L^{2}(\partial D)$ of the analytic polynomials, and define Toeplitz operators as usual. As in Section $1, T_{z} T_{\bar{z}}-T_{\bar{z}} T_{z}=T_{z} T_{\bar{z}}$ $-T_{|z|^{2}}$ is diagonal with eigenvectors $z^{n} w^{m}$. The eigenvalues corresponding to the vectors $\left\{w^{m}\right\}$ are

$$
\lambda(m)=-\frac{\left\|z w^{m}\right\|^{2}}{\left\|w^{m}\right\|^{2}}=\frac{\left[\frac{1}{2}+\frac{1}{m+1}\right]}{\left[1+\frac{1}{m+1}\right]},
$$

and since $\lambda(m) \rightarrow \frac{1}{2}$ as $m \rightarrow \infty$, the operator cannot be compact. (We point out that these Toeplitz operators are not the same as those defined on the distinguished boundary $S^{1} \times S^{1}$ of $D$, which have been extensively studied (see Douglas (1972b), Sections 9 and 10). However, the ones considered here are the natural analogue of those we looked at earlier: in fact, the polydisc can be thought of as a limiting case of the domains $\Omega$.)

\section{Acknowledgement}

We would like to thank John Loxton for help with the asymptotics in Section 1.

\section{References}

L. Boutet de Monvel (1979), 'On the index of Toeplitz operators of several complex variables', Invent. Math. 50, 249-272.

L. A. Coburn (1973), 'Singular integral operators and Toeplitz operators on odd spheres', Indiana Univ. Math. J. 23, 433-439. 
J. P. D'Angelo (1978), 'A note on the Bergmann kernel', Duke Math. J. 45, 259-265.

R. G. Douglas (1972a), Banach algebra techniques in operator theory (Academic Press, New York).

R. G. Douglas (1972b), Banach algebra techniques in the theory of Toeplitz operators (CMBS regional conference series in mathematics 15, Amer. Math. Soc., Providence).

A. Erdélyi (Editor) (1953), Higher transcendental functions, Vol. 1 (McGraw-Hill, New York).

G. B. Folland and J. J. Kohn (1972), The Neumann problem for the Cauchy-Riemann complex (Annals of Mathematics studies 75, Princeton University Press, Princeton, N. J.).

M. Hakim et N. Sibony (1977), 'Quelques conditions pour l'existence de fonctions pics dans des domaines pseudoconvexes', Duke Math. J. 44, 399-406.

J. Janas (1976a), 'Index formula for Toeplitz operators in certain strongly pseudoconvex domains', Bull. Acad. Polon. Sci. Sér. Sci. Math. Astronom. Phys. 24, 433-436.

J. Janas (1976b), 'An application of the theorem of Rudin to the Toeplitz operators on odd spheres', Math. Z. 150, 185-187.

N. P. Jewell and S. G. Krantz (1979), Toeplitz operators and related function algebras on certain pseudoconvex domains', Trans. Amer. Math. Soc. 252, 297-312.

I. Raeburn (1978), 'On Toeplitz operators associated with strongly pseudoconvex domains', Studia Math. 63, 253-258.

H. Sato and K. Yabuta (1978); 'Toeplitz operators on strongly pseudoconvex domains in Stein spaces', Tôhoku Math.J. 30, 153-162.

U. Venugopalkrishna (1972), 'Fredholm operators associated with strongly pseudoconvex domains', J. Functional Analysis 9, 349-373.

S. M. Webster (1979), 'Biholomorphic mappings and the Bergmann kernel off the diagonal', Invent. Math. 51, 155-169.

School of Mathematics

University of New South Wales

Post Office Box 1

Kensington, N.S.W. 2033

Australia 